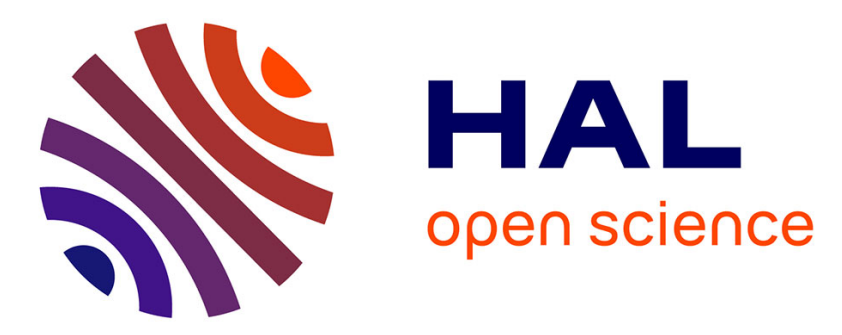

\title{
Réalisation d'un module de comptage de photons pour l'analyse de la lumière diffusée par les gels polymères
}

M. Ankrim, S.J. Candau

\section{To cite this version:}

M. Ankrim, S.J. Candau. Réalisation d'un module de comptage de photons pour l'analyse de la lumière diffusée par les gels polymères. Revue de Physique Appliquée, 1986, 21 (1), pp.65-71. 10.1051/rphysap:0198600210106500 . jpa-00245412

\section{HAL Id: jpa-00245412 https://hal.science/jpa-00245412}

Submitted on 1 Jan 1986

HAL is a multi-disciplinary open access archive for the deposit and dissemination of scientific research documents, whether they are published or not. The documents may come from teaching and research institutions in France or abroad, or from public or private research centers.
L'archive ouverte pluridisciplinaire HAL, est destinée au dépôt et à la diffusion de documents scientifiques de niveau recherche, publiés ou non, émanant des établissements d'enseignement et de recherche français ou étrangers, des laboratoires publics ou privés. 


\title{
Réalisation d'un module de comptage de photons pour l'analyse de la lumière diffusée par les gels polymères
}

\author{
M. Ankrim $\left({ }^{+}\right)$et S. J. Candau \\ Laboratoire de Spectrométrie et d'Imagerie Ultrasonores, Formation Associée au C.N.R.S., Université Louis \\ Pasteur, 4, rue Blaise Pascal, 67070 Strasbourg Cedex, France
}

(Recu le 30 novembre 1984, révisé le 19 septembre 1985, accepté le 20 septembre 1985)

\begin{abstract}
Résumé. - Cet article décrit le principe d'un compteur de photons numérique qui peut s’intégrer dans une chaîne de mesure de diffusion de lumière élastique et quasi élastique. Ce dispositif permet d'analyser en temps réel une intensité lumineuse caractérisée par une composante cohérente et une composante présentant des fluctuations temporelles. Des applications à l'étude de gels polymères sont présentées.
\end{abstract}

\begin{abstract}
This paper describes a digital photon counter which can be inserted in an elastic and quasi-elastic light scattering set-up. This apparatus allows to analyse in real time a scattered intensity characterized by a coherent component and a fluctuating one. Some applications to the study of polymer gels are presented.
\end{abstract}

\section{Introduction.}

Les techniques de diffusion élastique et quasi-élastique de la lumière sont couramment utilisées en laboratoire, principalement pour la caractérisation de hauts polymères ou de substances colloïdales en milieu très dilué. Les mesures de l'intensité diffusée et de la fonction d'autocorrélation temporelle correspondante fournissent en effet des informations sur la masse, la morphologie et la polydispersité des particules dissoutes ou en suspension.

$\mathrm{Au}$ cours de la dernière décade, l'emploi des techniques de diffusion de lumière s'est généralisé à des systèmes plus complexes tels que les solutions et suspensions semi-diluées ou concentrées et les gels.

Un problème qui a particulièrement attiré l'intérêt des chercheurs concerne l'étude des fluctuations de concentration coopératives appelées également « modes de gel » dans les systèmes formés soit par de longues chaînes macromoléculaires en solution semidiluée, soit par des réticulats gonflés [1,2]. Des difficultés ont cependant surgi, liées à la présence dans ces matériaux, principalement les réticulats, d'inhomogénéités de concentration à une échelle supramoléculaire [3-4]. Ces inhomogénéités, de taille supérieure à la longueur d'onde lumineuse, jouent le rôle de diffuseurs statiques ou quasi-statiques et fournissent

$\left(^{+}\right)$Adresse actuelle : Département de Physique, Université de Cadi Ayyad, Marrakech, Maroc. une contribution au signal lumineux diffusé qui se superpose à celle due aux fluctuations d'origine dynamique. Cet effet a également des incidences sur la fonction d'autocorrélation temporelle de l'intensité diffusée qui contient des termes de battements entre les deux composantes du signal. Il est donc nécessaire à la fois pour la mesure de l'intensité dynamique qui est généralement le paramètre d'intérêt et pour l'interprétation sans ambiguité de la fonction d'autocorrélation de pouvoir évaluer le rapport des intensités des deux composantes.

Ce rapport peut être obtenu au moyen d'un corrélateur numérique ou d'un analyseur de spectre $[5,6]$. Cependant, l'intensité diffusée totale présentant des dérives au cours du temps, vraisemblablement par suite de mouvements des inhomogénéités, il est nécessaire d'effectuer des mesures sur des intervalles de temps les plus courts possible. Une technique utilisant l'emploi d'un voltmètre efficace a été précédemment décrite [6]. Dans cet article nous présentons un dispositif numérique de comptage de photons permettant une étude statistique du nombre de photons détectés durant des intervalles de temps donnés. La détermination des moments du premier et du second ordre de la distribution du nombre de photons détectés pendant un temps d'échantillonnage fournit une mesure du rapport de l'intensité diffusée par les fluctuations dynamiques de concentration en polymère à l'intensité totale. Après avoir décrit le dispositif expérimental que nous avons réalisé, nous 
présentons quelques illustrations de son application à l'étude des gels.

\section{Principe de la méthode de mesure.}

La statistique des photoélectrons détectés est décrite par la loi de Mandel [7, 8]

$$
p(n, T)=\int_{0}^{\infty} \frac{(\alpha U)^{n}}{n !} \exp (-\alpha U) p(U) \mathrm{d} U
$$

où $p(n, T)$ est la densité de probabilité du nombre $n$ de photoélectrons détectés pendant un intervalle de temps $T$.

$p(U)$ est la densité de probabilité associée à l'intensité lumineuse diffusée $U$.

$\alpha$ est l'efficacité quantique du détecteur.

Généralement, la connaissance de la moyenne statistique $\langle n\rangle$ et de la variance $V(n)$ est suffisante pour décrire la statistique.

$$
\begin{gathered}
\langle n\rangle=\alpha\langle U\rangle \\
V(n)=\langle n\rangle+\alpha^{2} V(U)
\end{gathered}
$$

avec

$$
V(U)=\left\langle U^{2}\right\rangle-\langle U\rangle^{2} .
$$

Les détecteurs photoélectriques ne peuvent être considérés comme idéaux et il convient généralement de prendre en considération des effets liés à l'intégration spatiale et temporelle du signal détecté ainsi qu'au temps mort suivant l'enregistrement d'une impulsion. La statistique du signal peut également être modifiée par la présence d'impulsions retardées. Ces dernières cependant ne produisent un effet appréciable que pour des taux de comptage très faibles [13], ce qui n'est pas le cas dans les expériences présentes.

1.1 INTÉGRATION TEMPORELLE. - Dans le cas général où le temps d'échantillonnage $T$ n'est pas très petit ou très grand devant le temps de cohérence $T_{\mathrm{c}} \mathrm{du}$ signal détecté, la densité de probabilité $p(U)$ est donnée par la formule, non rigoureuse, suivante [9-13] :

$$
p(U)=\frac{a^{N}}{2^{N}} \frac{U^{N-1}}{(N-1) !} \mathrm{e}^{-\frac{a U}{2}}
$$

avec

$$
\begin{gathered}
a=\frac{2 N}{\langle U\rangle} \\
N=T^{2}\left[\int_{0}^{T} \int_{0}^{T}\left|\gamma\left(t-t^{\prime}\right)\right|^{2} \mathrm{~d} t \mathrm{~d} t^{\prime}\right]^{-1} \\
\gamma(\tau)=\Gamma(\tau) / \Gamma(0)
\end{gathered}
$$

et $\Gamma$ représente la fonction gamma.

A partir des expressions (1)-(4) on peut obtenir la densité de probabilité $p(n)$ et la variance $V(n)$

$$
\begin{gathered}
p(n)=\frac{\Gamma(N+n)}{n ! \Gamma(n)} \cdot \frac{1}{\left(1+\frac{\langle n\rangle}{N}\right)^{N}} \cdot \frac{1}{\left(1+\frac{N}{\langle n\rangle}\right)^{n}} \\
V(n)=\langle n\rangle\left(1+\frac{\langle n\rangle}{N}\right) .
\end{gathered}
$$

Lorsque le spectre du signal diffusé est Lorentzien, ce qui est généralement le cas, l'expression (6) peut s'écrire sous la forme [13]

$$
V(n)=\langle n\rangle[1+f(\delta)\langle n\rangle]
$$

avec :

$$
f(\delta)=\frac{1}{\delta}-\frac{1}{2 \delta^{2}}+\frac{1}{2 \delta^{2}} \mathrm{e}^{-2 \delta}
$$

et $\delta=T / T_{\mathrm{c}}$.

1.2 InTÉGRATION SPATIALE. - Les effets d'intégration spatiale du signal diffusé ont été analysés en détail par Jakeman et al. [13, 14].

Lorsque la cohérence spatiale est incomplète, la variance $V(n)$ s'écrit :

$$
V(n)=\langle n\rangle[1+\langle n\rangle f(\delta) f(A)] .
$$

Pour une source lumineuse circulaire de rayon $\rho$ (en l'occurrence le diaphragme entre le volume diffusant et le détecteur) et un détecteur circulaire de rayon $r$ (défini également par un diaphragme), $f(A)$ s'écrit :

$f(A)=\sum_{s=0}^{\infty}\left\{\frac{(2 s+2) !}{[(s+1) !]^{2}(s+2) !}\right\}^{2}(-1)^{s}\left(\frac{1}{2} \kappa r\right)^{2 s}$

avec $\kappa=k_{0} \rho / z$ où $k_{0}$ est le vecteur d'onde de la radiation lumineuse et $z$ la distance entre la source et le détecteur.

Trois cas présentent un intérêt particulier pour notre étude :

1) $T / T_{\mathrm{c}} \gg 1$. C'est le cas où le détecteur ne reçoit que de la lumière cohérente. L'équation (8) devient :

$$
V(n)=\langle n\rangle .
$$

2) $T / T_{c} \ll 1$. Aux fluctuations intrinsèques du processus de détection se superposent les fluctuations d'intensité lumineuse.

Dans ce cas, l'équation (8) devient :

$$
V(n)=\langle n\rangle[1+\langle n\rangle f(A)] .
$$

3) Le détecteur reçoit simultanément de la lumière cohérente d'intensité $\left\langle n_{\mathrm{c}}\right\rangle$ et un signal GaussienLorentzien (avec $\left.T / T_{\mathrm{c}} \ll 1\right)$ d'intensité $\left\langle n_{\mathrm{f}}\right\rangle$.

Dans ce cas, l'expression (11) peut se généraliser à $[6,15]$ :

$$
V(n)=\langle n\rangle\left[1+f(A)\left\langle n_{\mathrm{f}}\right\rangle\left(1+\frac{\left\langle n_{\mathrm{c}}\right\rangle}{\langle n\rangle}\right)\right]
$$


où

$$
\langle n\rangle=\left\langle n_{\mathrm{c}}\right\rangle+\left\langle n_{\mathrm{f}}\right\rangle \text {. }
$$

1.3 EFFETS DE TEMPS MORT. - Après chaque enregistrement d'une impulsion le détecteur ne répond plus à un champ externe pendant un temps $\tau_{\mathbf{D}}$. De ce fait, le nombre d'impulsions enregistrées pendant une durée $T$ sera inférieur au nombre réel d'impulsions et les distributions mesurées de photoélectrons seront modifiées. Cet effet a été étudié en détail par Johnson et al. [16] et Bedard [17].

Le calcul des moments des premier et deuxième ordres effectué à partir de la densité de probabilité modifiée pour tenir compte du temps mort dans l'hypothèse $\tau_{\mathrm{D}} \ll T$ conduit à [15] :

$$
\begin{gathered}
\left\langle n_{\text {exp }}\right\rangle=\langle n\rangle-\frac{\tau_{\mathrm{D}}}{T}\langle n\rangle^{2}\left(1+\frac{1}{N}\right) \\
\left\langle n_{\text {exp }}\left(n_{\text {exp }}-1\right)\right\rangle=\langle n\rangle^{2}+\frac{\langle n\rangle^{2}}{N}-\frac{2 \tau_{\mathrm{D}}}{T} \\
\times\left[\langle n\rangle^{2}+\frac{\langle n\rangle^{2}}{N}+\langle n\rangle^{3}+\frac{\langle n\rangle^{3}}{N}\left(3+\frac{2}{N}\right)\right]
\end{gathered}
$$

où $n_{\exp }$ représente le nombre de photoélectrons mesurés.

Dans les cas limites où $T$ est beaucoup plus grand ou plus petit que $T_{\mathrm{c}}$ les deux expressions ci-dessus deviennent :

$T / T_{\mathrm{c}} \gg 1$

$\left\langle n_{\exp }\right\rangle=\langle n\rangle-\langle n\rangle^{2}\left(\frac{\tau_{\mathrm{D}}}{T}\right)$

$\left\langle n_{\exp }\left(n_{\exp }-1\right)\right\rangle=\langle n\rangle^{2}-2\left(\frac{\tau_{\mathrm{D}}}{T}\right)\left(\langle n\rangle^{2}+\langle n\rangle^{3}\right)$

$T / T_{\mathrm{c}} \ll 1$

$\left\langle n_{\text {exp }}\right\rangle=\langle n\rangle-2\langle n\rangle^{2}\left(\frac{\tau_{\mathrm{D}}}{T}\right)$

$\left\langle n_{\exp }\left(n_{\exp }-1\right)\right\rangle=2\langle n\rangle^{2}-4\left(\frac{\tau_{\mathrm{D}}}{T}\right)\left(\langle n\rangle^{2}+3\langle n\rangle^{3}\right)$.

\section{Dispositif expérimental.}

La figure 1 montre comment le module de comptage de photons que nous avons réalisé, s'intègre dans la chaîne de mesure de lumière diffusée. La composante principale du module est un microprocesseur $280 \mathrm{~A}$ qui gère les autres fonctions (compteur, roulette, interface de dialogue avec l'extérieur, afficheurs, bouton poussoir et interrupteur). Le module a pour tâche essentielle de faire l'acquisition de 10240 échantillons de durée ajustable. Il peut enregistrer jusqu'à 16 impulsions par temps d'échantillonnage. Ce dernier peut être ajusté entre $1 \mu$ s et $100 \mu$ s. Dans les expériences présentées ci-dessous il est fixé à $1 \mu \mathrm{s}$. Le traitement informatique de ces échantillons conduit

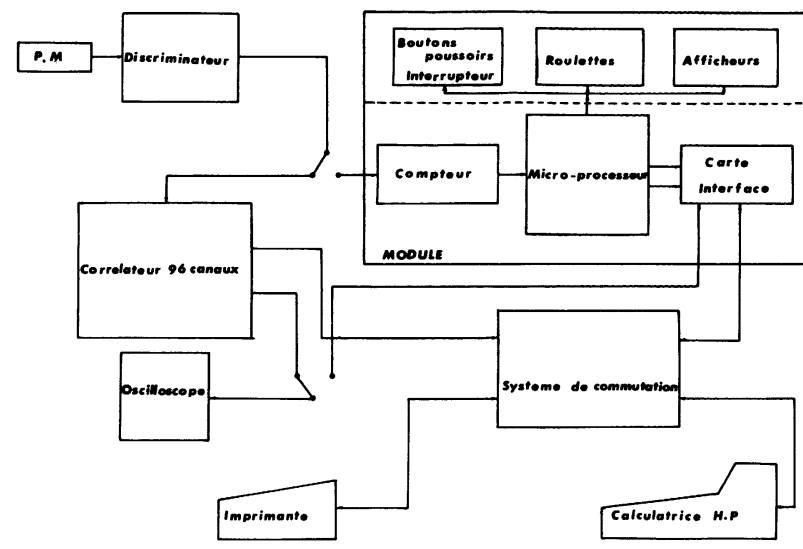

Fig. 1. - Schéma de la chaîne de mesures.

[Schematic diagram of the set-up.]

à la détermination des moyennes statistiques $\left\langle n_{\exp }\right\rangle$ et $V\left(n_{\text {exp }}\right)$. Ces quantités sont alors corrigées en utilisant le système d'équations (15) pour fournir $\langle n\rangle$ et $V(n)$. La correction s'effectue en temps réel, point par point, en utilisant pour $\tau_{\mathbf{D}}$ la valeur de $55 \mathrm{~ns}$, mesurée expérimentalement à partir de la réponse du discriminateur à un signal impulsionnel de périodicité variable. Cette valeur de $\tau_{\mathbf{D}}$ est en bon accord avec les données techniques de l'appareil.

La partie électronique du module comporte :

- une carte d'acquisition et de comptage d'impulsions,

- une carte microprocesseur et mémoire,

- une interface de dialogue avec l'extérieur.

En ce qui concerne la partie informatique, le module dispose de $16 \mathrm{~K}$-octets de programme et de $4 \mathrm{~K}$-octets de mémoire de travail qui permettent de réaliser les programmes suivants :

- Traitement des échantillons

- Gestion des périphériques et dialogue avec l'opérateur

- Visualisation

- Gestion des erreurs

- Mise au point.

L'organigramme du fonctionnement global du module est représenté sur la figure 2 . On peut remarquer que le module peut fonctionner soit en mode autonome, soit en mode commandé par la calculatrice de type HP.

\section{Tests expérimentaux.}

Nous avons contrôlé le bon fonctionnement du module pour les trois situations expérimentales correspondant aux trois cas mentionnés dans le paragraphe 1.

3. 1 LA LUMIÈRE REÇUE PAR LE DÉTECTEUR EST COHÉRENTE $\left(T / T_{\mathrm{c}} \gg 1\right)$. - Nous avons vu que dans ce 


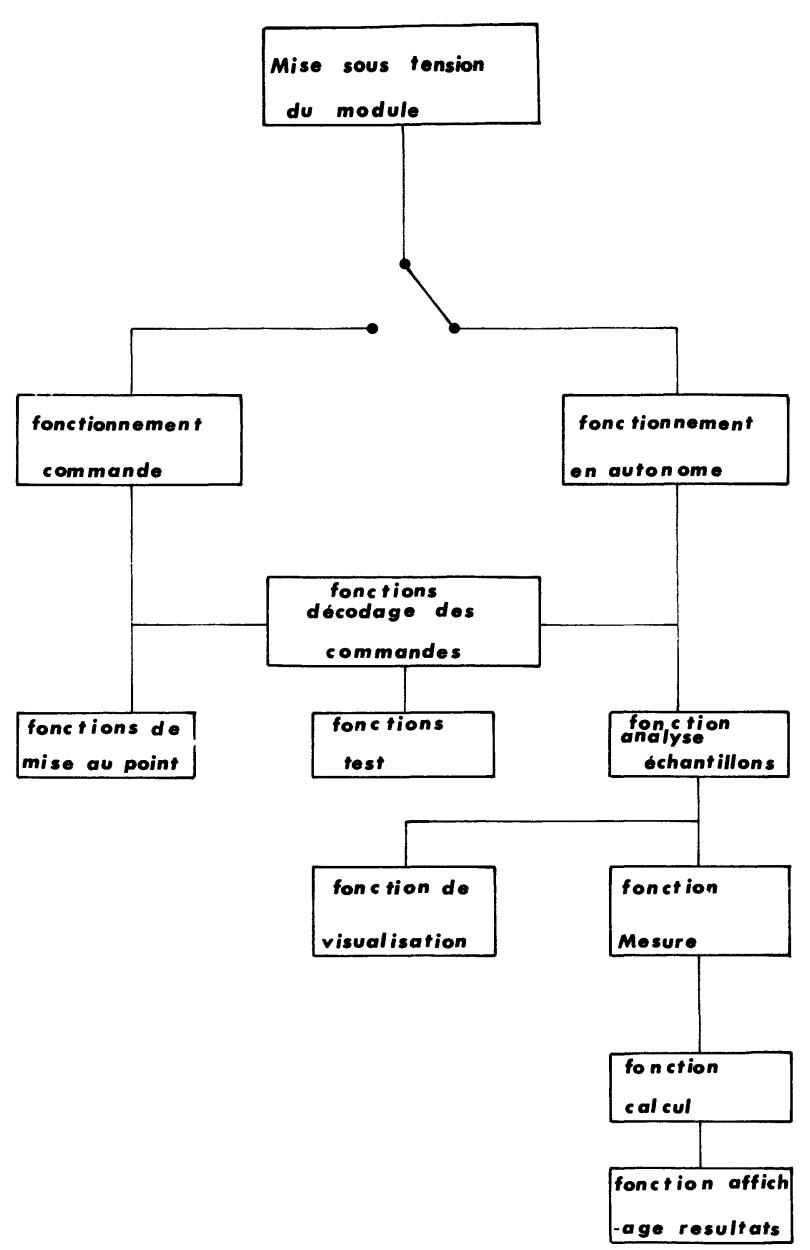

Fig. 2. - Organigramme du fonctionnement du module.

[Functional diagram of the photocounter.]

cas le rapport $R=V(n) /\langle n\rangle$ est indépendant de $\langle n\rangle$. La figure 3 montre les résultats pour des expériences réalisées avec un laser à argon ionisé $(\lambda=488 \mathrm{~nm})$ et un photomultiplicateur ITT FW 130. Les valeurs de $R$ ont été obtenues après correction des effets de temps mort d'après le système d'équations (14). Sur la même figure nous avons reporté les résultats obtenus sans effectuer la correction pour le temps mort. La décroissance observée de $R$ lorsque $\left\langle n_{\exp }\right\rangle$ augmente illustre l'importance de cet effet.

3.2 LA LUMIÈre REÇUE PAR LE DÉTECTEUR PRÉSENTE UN SPECTRE LORENTZIEN $\left(T / T_{\mathrm{c}} \ll 1\right)$. - L'expérience a été réalisée en utilisant comme diffuseur une solution de polystyrène de masse moléculaire $M_{\mathrm{w}}=10^{6}$ dans le cyclopentane, à une concentration de $0,4 \%$. Pour cette solution, $T_{\mathrm{c}}$, déterminé à partir de la mesure de la fonction d'autocorrélation est égal à $38 \mu$ s à $25^{\circ} \mathrm{C}$ et pour un angle de diffusion $\theta=90^{\circ}$.

Dans cette expérience, la surface utile de la cathode du photomultiplicateur est limitée par le rayon de l'ouverture d'un diaphragme; $r=50 \mu \mathrm{m}$. La distance $z$ est égale à $12 \mathrm{~cm}$. Le rayon de la source est défini par

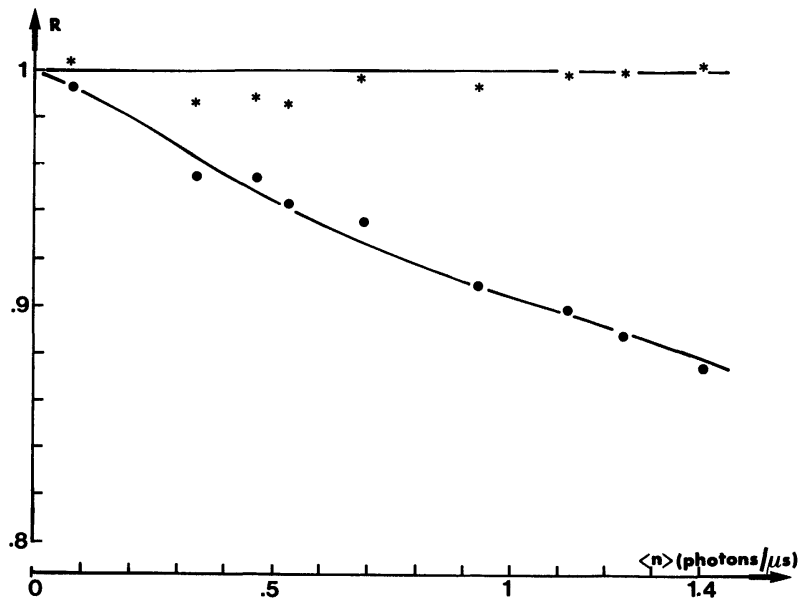

Fig. 3. - Variation du paramètre $R$ en fonction de l'intensité lumineuse moyenne du faisceau laser; $\tau_{\mathrm{D}}=55 \mathrm{~ns}$. Sans correction de temps mort, * Avec correction.

[Variation of parameter $R$ as a function of the average scattered intensity of the incident light; $\tau_{\mathrm{D}}=55 \mathrm{~ns}$. - Raw data, * Data corrected for dead time effect.]

l'ouverture $\rho$ d'un diaphragme variable. Le tableau illustre la comparaison pour 2 ouvertures du diaphragme entre les valeurs de $f(A)$ calculées à partir de l'équation (9) et les valeurs obtenues expérimentalement à partir de $R$ (Eq. (11)). On observe un bon accord à condition de tenir compte de la correction de temps mort.

Tableau. - Comparaison entre les valeurs calculées et expérimentales de $f(A)$.

$$
\begin{array}{ccc}
\rho(\mathrm{mm}) & 0,4 & 0,8 \\
f(A) \text { expérimental } & 0,69(0,51)\left({ }^{\mathrm{a}}\right) & 0,29(0,18)\left({ }^{\mathrm{a}}\right) \\
f(A) \text { calculé } & 0,7 & 0,32
\end{array}
$$

(a) Les valeurs entre parenthèses ont été obtenues en négligeant la correction de temps mort.

3.3 LE DÉTECTEUR REÇOIT SIMULTANÉMENT DE LA LUMIÈRE COHÉRENTE ET DE LA LUMIÈRE À SPECTRE LORENTZIEN. - Expérimentalement, cette situation est réalisée en envoyant simultanément sur la cathode du photomultiplicateur, le signal diffusé par la solution de polystyrène et une fraction du faisceau laser incident (montage dit « hétérodyne » [13]). L'intensité du faisceau cohérent peut être ajustée au moyen d'un atténuateur. On définit un rapport d'hétérodynage $r_{\mathrm{h}}$ :

$$
r_{\mathrm{h}}=\frac{\left\langle n_{\mathrm{c}}\right\rangle}{\left\langle n_{\mathrm{f}}\right\rangle}
$$

Dans une telle expérience $R$ est donné par l'équation (12). Si on norme par rapport à la valeur obtenue 
en l'absence de faisceau cohérent (régime homodyne), on obtient :

$$
\frac{\tilde{R}}{\tilde{R}_{0}}=\frac{[(R-1) /\langle n\rangle]_{\text {het }}}{[(R-1) /\langle n\rangle]_{\text {hom }}}=\frac{\left\langle n_{\mathrm{f}}\right\rangle}{\langle n\rangle}\left(1+\frac{\left\langle n_{\mathrm{c}}\right\rangle}{\langle n\rangle}\right)
$$

ou encore :

$$
\frac{\tilde{R}}{\widetilde{R}_{0}}=\frac{1}{1+r_{\mathrm{h}}}\left(1+\frac{1}{1+r_{\mathrm{h}}^{-1}}\right) .
$$

Les moyennes $\left\langle n_{\mathrm{f}}\right\rangle$ et $\left\langle n_{\mathrm{c}}\right\rangle$ pouvant être déterminées indépendamment, il est possible de confronter les valeurs expérimentales de $\tilde{R} / \tilde{R}_{0}$ à celles calculées à partir de l'équation (17).

Cette comparaison est illustrée sur la figure 4 où le paramètre $\tilde{R} / \widetilde{R}_{0}$ est porté en fonction de $r_{\mathrm{h}}$. Le désaccord observé pour les valeurs intermédiaires de $r_{\mathrm{h}}$ peut être imputé à la correction de temps mort. Celle-ci est effectuée en résolvant le système d'équations (13) de manière à obtenir $\langle n\rangle$ et $N$. Cette procédure ne constitue cependant qu'une approximation, la correction de temps mort ne devenant rigoureuse que pour $r_{\mathrm{h}} \simeq 1$ et $r_{\mathrm{h}}>\sim 10$.

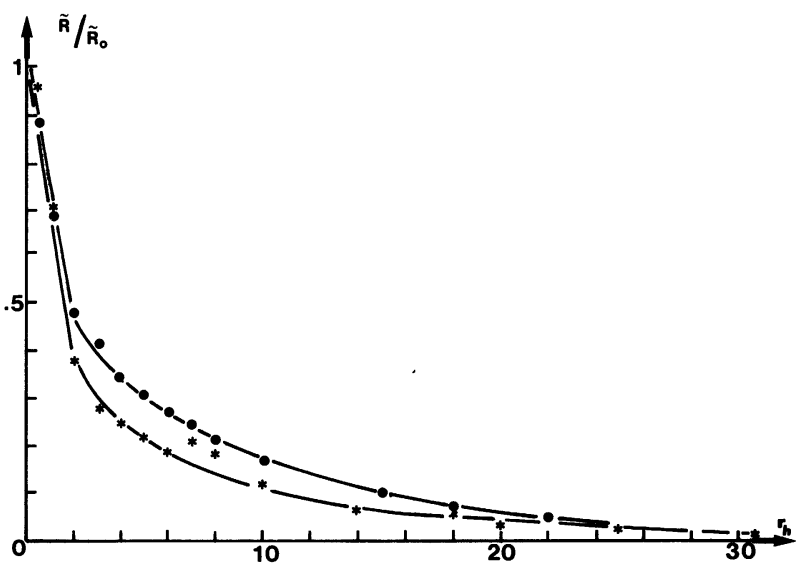

Fig. 4. - Variation de $\tilde{R} / \tilde{R}_{0}$ en fonction du rapport d'hétérodynage $r_{\mathrm{h}}$ * Valeurs expérimentales, $\bullet$ Valeurs calculées à partir de l'équation (17).

[Variation of $\tilde{R} / \tilde{R}_{0}$ as a function of heterodyne ratio $r_{\mathrm{h}}$. * Experimental values, - Values calculated from equation (17).]

De ce fait, pour les applications on utilisera de préférence la courbe expérimentale de $\widetilde{R} / \widetilde{R}_{0}$ en fonction de $r_{\mathrm{h}}$ qui servira de courbe d'étalonnage.

\section{Applications à l'étude des inhomogénéités dans les gels polymères.}

La présence dans les réticulats polymères gonflés, d'inhomogénéités de structure, à des échelles très supérieures à la maille du réticulat, a été mise en évidence au moyen de différentes techniques
[3-6, 18-22]. Dans une expérience de diffusion de lumière, les inhomogénéités qui sont quasi statiques diffusent un faisceau cohérent dont l'intensité (ramenée à volume diffusant constant) est susceptible de varier
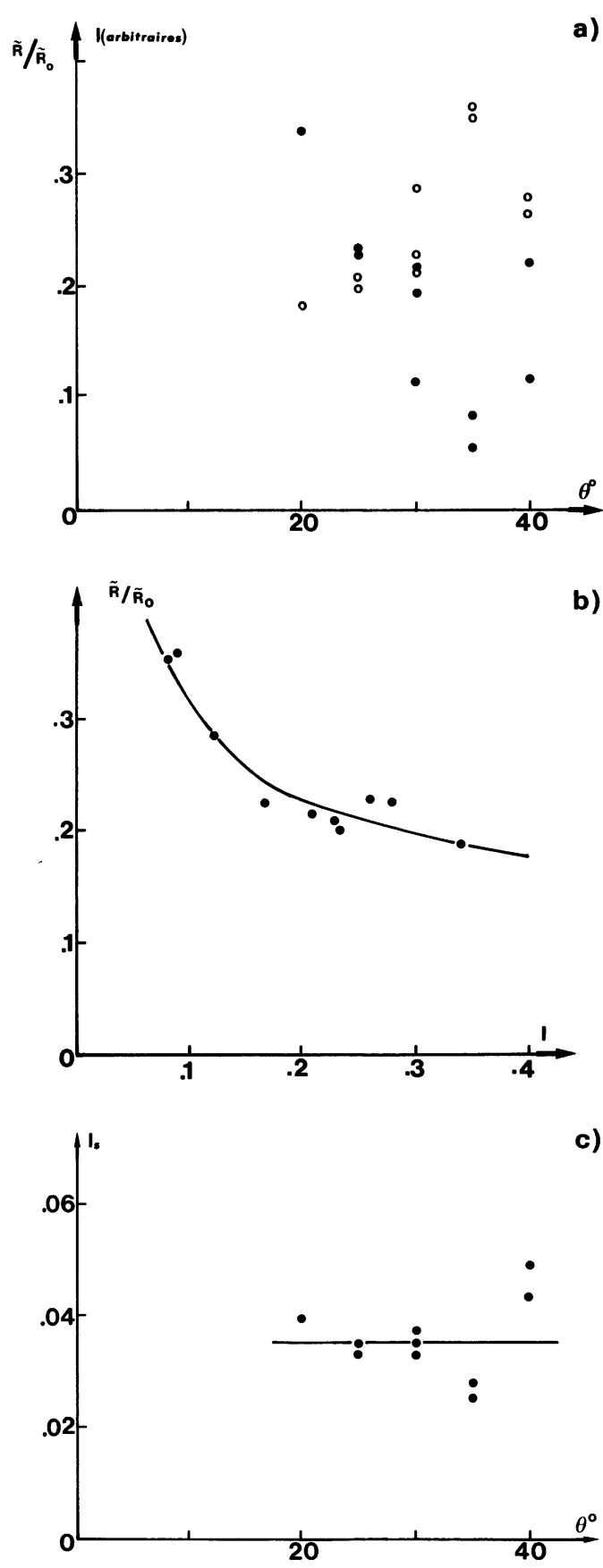

Fig. 5. - Gel de polystyrène-toluène. a) Variation de : $\bigcirc \tilde{R} / \tilde{R}_{0}$ et $\bullet$ Intensité en fonction de l'angle de diffusion $\theta$, b) Variation de $\tilde{R} / \tilde{R}_{0}$ en fonction de $I$, c) Variation de l'intensité diffusée associée aux fluctuations dynamiques de concentration en fonction de $\theta$. Les intensités sont données en unités arbitraires.

[Polystyrene-toluene gel. a) Variation of : $\widetilde{R} / \widetilde{R}_{0}$ and $\bullet$ Intensity as a function of scattering angle $\theta, \mathrm{b}$ ) Variation of $\tilde{R} / \tilde{R}_{0}$ as a function of $I, \mathrm{c}$ ) Variation of dynamic scattered intensity as a function of $\theta$.] 
avec l'angle de diffusion et la place du faisceau dans l'échantillon. A cette contribution vient s'ajouter la lumière diffusée par les fluctuations dynamiques qui est indépendante de l'angle de diffusion $[2,6,23]$.

Cette situation est tout à fait comparable à celle décrite dans le paragraphe précédent, le signal associé aux inhomogénéités jouant le rôle d'un oscillateur local qui hétérodyne l'intensité fluctuante. Le module de comptage de photons décrit dans cet article permet de mesurer le rapport des 2 contributions, autrement dit le rapport d'hétérodynage $r_{\mathrm{h}}$.

Pour illustrer cette application, nous avons choisi deux systèmes : un réticulat de polyacrylamide préparé par voie radicalaire et gonflé par de l'eau (fraction volumique du polymère $5 \%$ ), et un réticulat de polystyrène préparé par irradiation $\gamma$ et gonflé par du toluène [24] (fraction volumique du polymère $6 \%$ ).

La figure $5 \mathrm{a}$ représente les variations en fonction de l'angle de diffusion $\theta$ de l'intensité diffusée $I$ (après
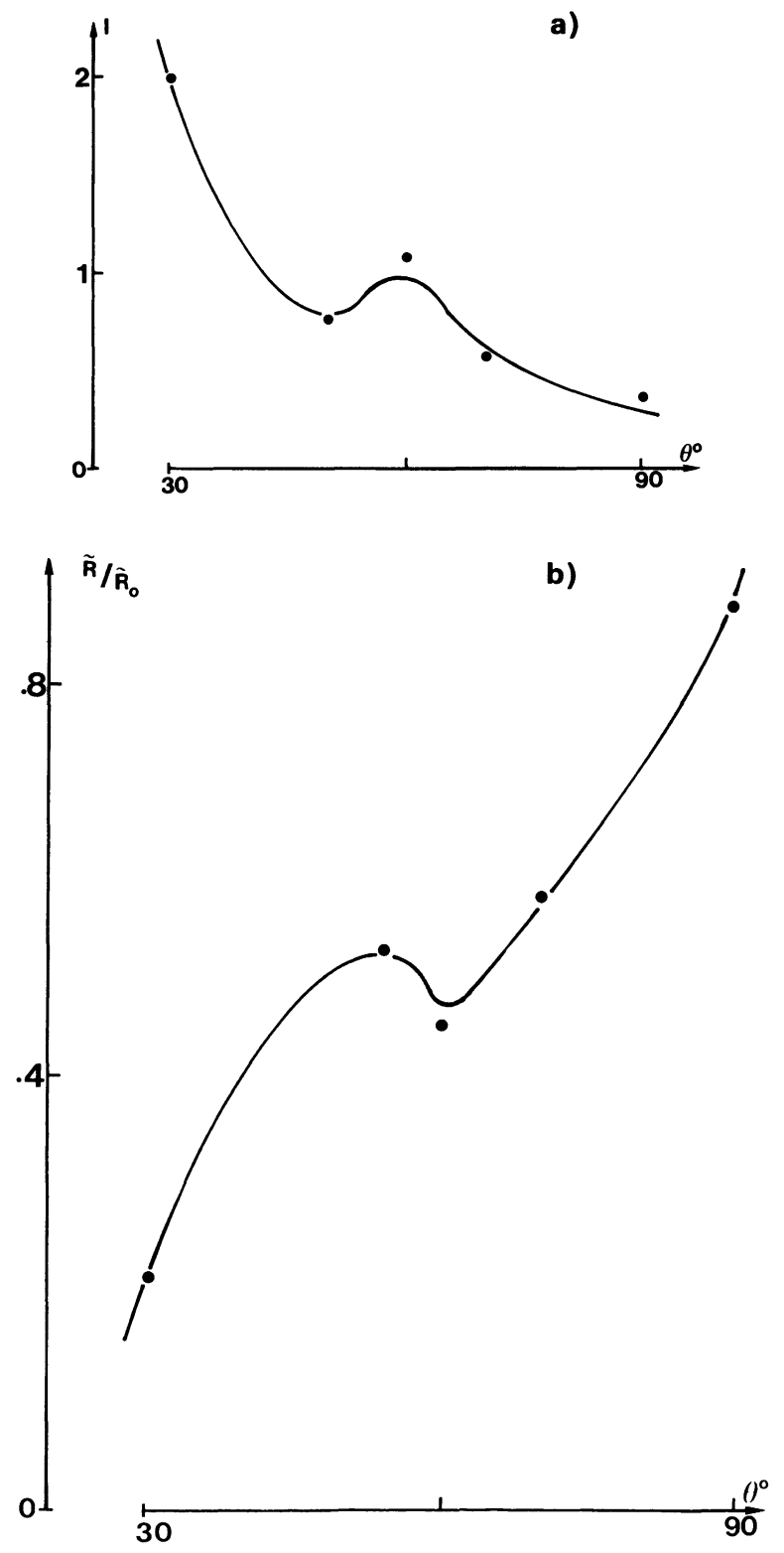

correction pour le volume diffusant) et du paramètre $\widetilde{R} / \widetilde{R}_{0}$ pour le gel de polystyrène. La valeur de $\widetilde{R}_{0}$ a été déterminée au moyen d'une mesure dans la solution étalon de polystyrène (cf. paragraphe 3) en conservant la même géométrie expérimentale. Les variations de $I$ et $\widetilde{R} / \widetilde{R}_{0}$ sont assez aléatoires en fonction de $\theta$ mais sont corrélées entre elles comme l'illustre la figure $5 b$. En effet, la courbe monotone obtenue pour la variation de $\widetilde{R} / \widetilde{R}_{0}$ en fonction de $I$ traduit bien que les variations d'intensité diffusée proviennent de la composante statique. A partir de la courbe de conversion de la figure 4, on peut déduire le rapport d'hétérodynage et par suite, l'intensité diffusée moyenne $I_{\mathrm{s}}$ due aux fluctuations dynamiques (Fig. 5c). Cette dernière est constante aux erreurs près. La séquence des figures 6(a-b-c-d) illustre le même phénomène pour le gel de polyacrylamide. De nouveau, la forme de la courbe $I(\theta)$ n'a pas de signification physique réelle, car elle dépend de l'emplacement

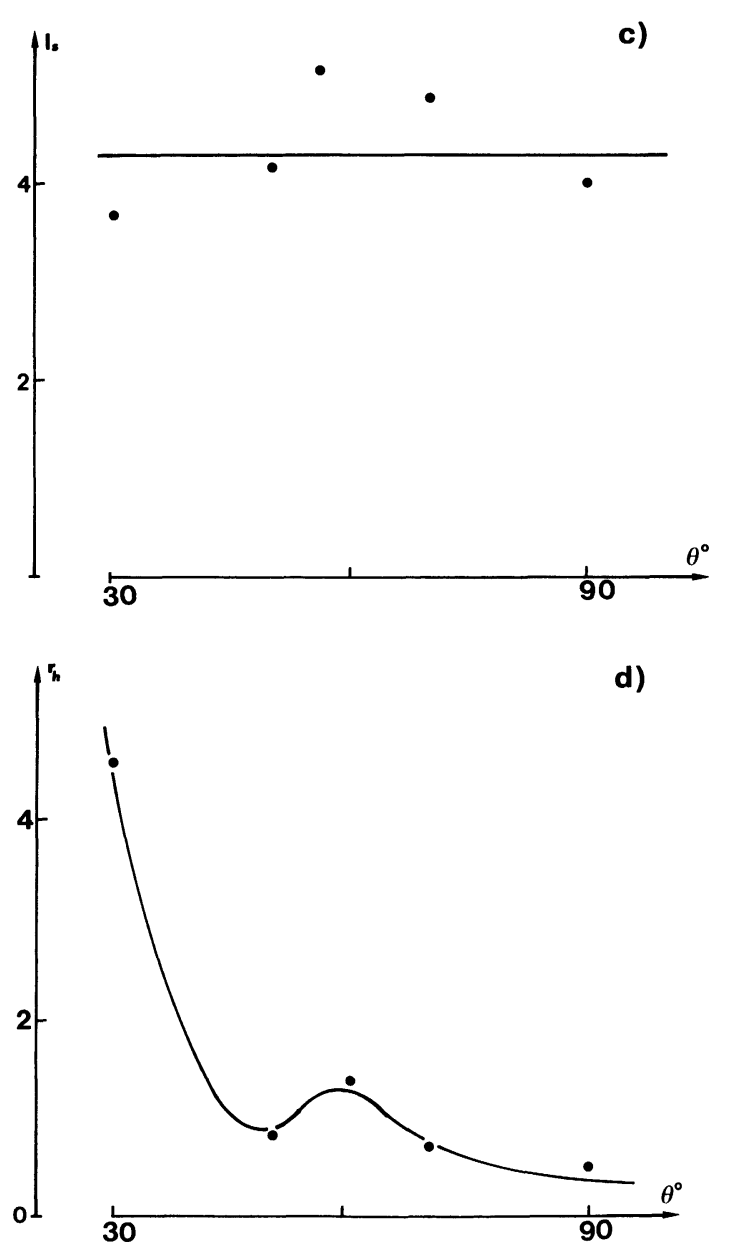

Fig. 6. - Gel de polyacrylamide-eau. Les variations en fonction de $\theta$, de $I, \tilde{R} / \tilde{R}_{0}, I_{\mathrm{s}}$ et $r_{\mathrm{h}}$ sont reportées respectivement sur les figures a, b, c, d. Les intensités sont données en unités arbitraires.

[Polyacrylamide-water gel. The variations of $I, \tilde{R} / \tilde{R_{0}}$, $I_{\mathrm{s}}$, and $r_{\mathrm{h}}$ as a function of $\theta$ are reported respectively on figures $a, b, c$ and $d$.] 
du gel, traversé par le faisceau laser. Il est par contre significatif que la variation de $\widetilde{R} / \widetilde{R}_{0}$ reproduise celle de $I$, montrant ainsi l'effet des hétérogénéités dans le gel. L'intensité $I_{\mathrm{s}}$ associée aux fluctuations dynamiques est, aux erreurs expérimentales près, indépendante de l'angle de diffusion comme le prévoit la théorie [2].

La précision sur $I_{\mathrm{s}}$ pour les gels peut être estimée à $\pm 15 \%$.

\section{Conclusion.}

Le dispositif numérique de comptage de photons décrit dans cet article permet d'étudier en temps réel des systèmes diffusant simultanément une lumière cohérente et une lumière présentant des fluctuations d'intensité gaussiennes-lorentziennes. Il permet de mesurer le rapport des deux contributions respectives. La détermination absolue de l'intensité associée aux fluctuations peut être réalisée au moyen d'un étalonnage avec un liquide tel que le benzène, de rapport de Rayleigh connu. Ce dispositif permet d'envisager des études intéressantes dans le domaine des gels polymères.

\section{Remerciements.}

Les auteurs remercient Mme Mahr, MM. Waton et Trio pour leur collaboration dans la mise au point des programmes et MM. Bastide et Mallo pour leur avoir fourni les gels utilisés dans cette étude.

\section{Bibliographie}

[1] De Gennes, P.-G., Scaling concepts in polymer physics (Cornell University Press, Ithaca, N.Y.) 1979.

[2] Candau, S., Bastide, J. et Delsanti, M., Adv. Polvm. Sci. 44 (1982) 27.

[3] Weiss, N., van Vlier, T. et Silberberg, J., Polym. Sci. 17 (1979) 2229.

[4] Prins, W., dans Polymer Networks, Ed. Chompff, T. (Plenum Press, N.Y.) (1971) p. 323.

[5] Geissler, E. et Hecht, A. M., J. Chem. Phys. 65 (1976) 103.

[6] Candau, S., Young, C. Y., Tanaka, T., Lemaréchal, P. et BAstide, J., J. Chem. Phys. 70 (1979) 4694.

[7] Mandel, L. dans Progress in Optics, Ed. Wolf, E. (North-Holland Publishing Co, Amsterdam) 1963, vol. II, p. 181.

[8] Mandel, L., Proc. Phys. Soc. 8 (1963) 1104.

[9] Rice, S. O., Bell System. Techn. J. 23 (1944) 282.

[10] Mandel, L., Proc. Phys. Soc. 74 (1959) 233.

[11] Troup, G. J., Proc. Phys. Soc. 86 (1964) 39.

[12] Bedard, G., Chang, J. C. et Mandel, L., Phys. Rev. 160 (1967) 1496.
[13] Photon correlation and light beating spectroscopy, Ed. Cummins, H. Z. et Pike, E. R. (Plenum Press, N.Y.) 1974.

[14] Jakeman, E., Oliver, C. J. et Pike, E. R., J. Phys. A 3 (1970) L45.

[15] ANkrim, M., Thèse Strasbourg, 1984.

[16] Johnson, F. A., Jones, R., McLean, T. P. et Pike, E. R., Phys. Rev. Lett. 16 (1966) 589.

[17] Bedard, G., Phys. Rev. 161 (1967) 1304.

[18] RuChel, R., STEEVE, R. L. et ERB, E. F., J. Chromato. 166 (1978) 563.

[19] Ruchel, R. et Brager, M. D., Anal. Biochem. 68 (1978) 415.

[20] Gupta, M. K. et Bansil, R., J. Polym. Sci. 19 (1981) 313.

[21] BansiL, R. et Gupta, M. K., Ferroelectrics 30 (1980) 63.

[22] Gupta, M. K. et Bansil, R., Polymer Reprint 22 (1982) 192.

[23] Munch, J. P., Candau, S. J., Herz, J. et Hild, G., J. Physique 38 (1977) 971. 